\title{
A rapid and simple method to detect ESBL in Enterobacter cloacae based on MIC of cefepime
}

\author{
Marina de Souza Bastos ${ }^{[1]}$, Thatiany Cevallos Menegucci ${ }^{[1]}$, \\ Rafael Renato Brondani Moreira ${ }^{[1]}$, Lourdes Botelho Garcia ${ }^{[1]}$, \\ Celso Luiz Cardoso ${ }^{[1]}$ and Maria Cristina Bronharo Tognim ${ }^{[1]}$
}

[1]. Departamento de Ciências Básicas da Saúde, Universidade Estadual de Maringá, Maringá, Paraná, Brasil.

\begin{abstract}
Introduction: The aim of this study was to identify a rapid and simple phenotypic method for extended-spectrum $\beta$-lactamase (ESBL) detection in Enterobacter cloacae. Methods: A total of 79 consecutive, non-repeated samples of E. cloacae were evaluated. Four phenotypic methods were applied for ESBL detection, results were compared to multiplex polymerase chain reaction (PCR) as the gold standard reference method: 1) ceftazidime and cefotaxime disks with and without clavulanate, both with boronic acid added; 2) disk approximation using cefepime and amoxicillin/clavulanate; 3) ESBL screening by minimum inhibitory concentration (MIC) $\geq 16 \mu \mathrm{g} / \mathrm{mL}$ and 4 ) by $\mathrm{MIC} \geq 2 \mu \mathrm{g} / \mathrm{mL}$ for cefepime. Results: Method 4 showed the best combination of sensitivity (100\%) and specificity (94\%). Conclusions: MIC $\geq 2 \mu \mathrm{g} / \mathrm{mL}$ for cefepime would be very useful for the phenotypic detection of ESBL in samples of E. cloacae.
\end{abstract}

Keywords: Enterobacter cloacae. ESBL. Phenotypic detection.

Enterobacter spp. are important opportunistic pathogens known to cause hospital acquired infections. A major cause of resistance to third-generation cephalosporins by Enterobacter spp. is the expression of AmpC-type $\beta$-lactamase. In addition, resistance in many clinical samples is due to the presence of conjugative plasmids encoding the gene extended-spectrum $\beta$-lactamase (ESBL) ${ }^{(1)}$.

Until the late 1990s, most ESBLs detected belonged to either the Temoniera (TEM) or sulfhydryl variable (SHV) types. More recently, the cefotaximases (CTX-M) type has rapidly spread and has been detected in samples from various countries ${ }^{(2)}$. The presence and types of ESBLs in Brazil, however, has not been well studied. We previously reported that the frequency of ESBL in Enterobacter spp. isolates was higher than was observed in both Escherichia coli and Klebsiella spp $^{(3)}$.

The standard tests for ESBL detection were developed by the Clinical and Laboratory Standards Institute (CLSI), and are based on the ability of clavulanic acid (CA) to inhibit the enzyme. However, this test is complicated by the presence of AmpC in Enterobacter spp. isolates, because CA also causes induction of $\mathrm{AmpC}^{(1)}$. To overcome $\beta$-lactamase interference in

Corresponding author: Dra. Maria Cristina Bronharo Tognim. Laboratório de Microbiologia/DBS/UEM. Av. Colombo 5790, 87020-900 Maringá, Paraná, Brasil.

Phone: 5544 3261-4952; Fax: 5544 3261-4860

e-mail: mcbtognim@uem.br

Received 2 October 2014

Accepted 28 November 2014
ESBL detection, the use of AmpC inhibitors has been proposed. However, the proposed methods have proved difficult to implement in routine laboratory testing. The aim of the present study was to identify the best and simplest phenotypic method for ESBL detection in clinical samples of Enterobacter cloacae.

This prospective study was conducted over a period of two years (2009-2011) in a 240-bed tertiary care hospital in Brazil. We used a total of 79 consecutive, non-repeated samples of E. cloacae recovered from clinical samples, including urine (44\%), blood (21\%), secretions (19\%), and colonization swabs $(16 \%)$. E. cloacae isolates were obtained primarily from patients in a 24-bed adult intensive care unit. All samples, belonging to 55 clones, were typed by the enterobacterial repetitive intergenic consensus-polymerase chain reaction (ERIC-PCR) molecular technique ${ }^{(4)}$. Isolates were judged to belong to the same genetic clone if the Dice correlation coefficient was 0.93 or greater. In this study we examined all independent isolates, because samples judged to belong to the same clone by ERIC-PCR may have different sensitivity profiles and show different phenotypic test results. Additionally, there is no standard procedure for selecting which isolates could be considered representative of their clonal groups for further analysis (Figure 1).

The identification and tests for antimicrobial sensitivity of isolates were carried out using an automated system (BD Phoenix ${ }^{\mathrm{TM}}$, Becton, Dickinson and Company, Sparks, MD, USA). Minimum inhibitory concentrations (MIC) of ceftazidime, ceftriaxone, and cefepime were assessed by the agar dilution method ${ }^{(5)}$ in Mueller Hinton Agar (Becton, Dickinson and Company) plates. Escherichia coli ATCC 25922 and Pseudomonas aeruginosa ATCC 27853 were used as controls. 


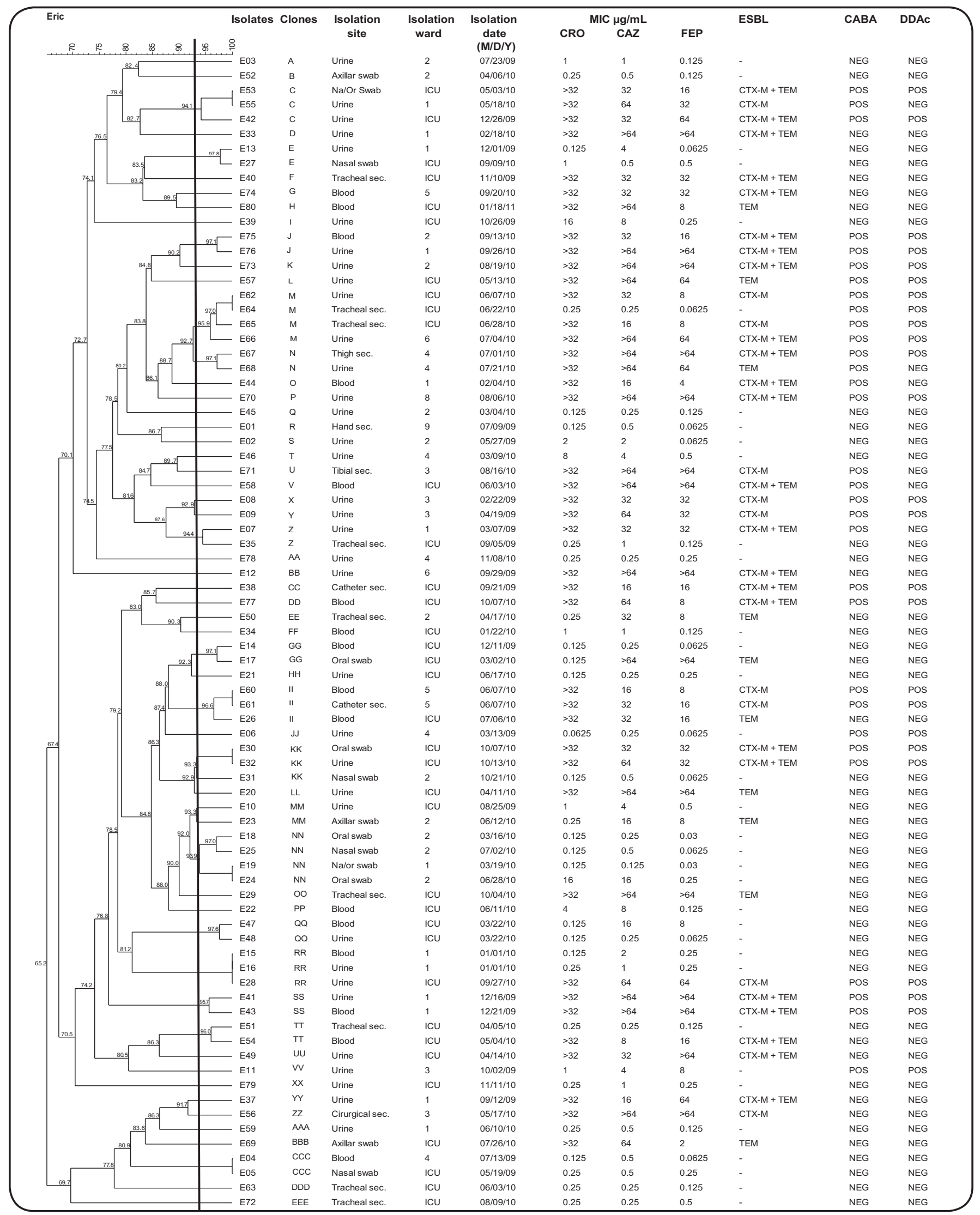

FIGURE 1 - Dendrogram plot obtained by the enterobacterial repetitive intergenic consensus-polymerase chain reaction (ERIC-PCR) analysis of the ESBL isolates. Hospital wards: ICU: intensive care unit; 1, 3, 5, and 8: clinical and surgical patients, differing only in the number of patients/room; 2: SUS (Brazilian National Unified Health System) patients; 4: emergency care; 6: maternity; 9: surgical center. Na/Or swab: nasal or oral swab; MIC: minimum inhibitory concentration; CRO: ceftriaxone; CAZ: ceftazidime; FEP: cefepime; ESBL: extended spectrum $\beta$-lactamase; CABA: ceftazidime and cefotaxime disks with and without clavulanic acid + boronic acid; DDAc: disk approximation using cefepime and amoxicillin/CA; CA: clavulanic acid; CTX-M: cefotaximases; TEM: Temoniera; NEG: negative; POS: positive. 
For detection and typing of ESBL, we performed multiplex PCR with primers for identification of the predominant ESBL types (TEM, SHV, and CTX-M), as described ${ }^{(6)}$. We performed four assays of phenotypic detection for ESBL activity. The first was ceftazidime and cefotaxime disks, with and without CA $(10 \mu \mathrm{g} / \mathrm{disk})$ added of $20 \mu \mathrm{L}$ of boronic acid (BA) solution at a concentration of $20 \mathrm{~g} / \mathrm{L}$ (Sigma, St. Louis, MO, USA), in which an increase $\geq 5 \mathrm{~mm}$ in the diameter of the halo surrounding the disk containing the drug $+\mathrm{CA}+\mathrm{BA}$ compared to the disk containing only the drug + BA was considered a positive result for $\mathrm{ESBL}^{(7)}$ (CABA method). The second assay was disk approximation using cefepime and amoxicillin/CA positioned at a distance of $20 \mathrm{~mm}$ center to center, in which the presence of a ghost zone (synergism) between the disks was considered a positive result for ESBL activity ${ }^{(8)}$ (DDAc method). The final two tests were a cefepime 16 (CPM16 method) and cefepime 2 (CPM2 method) assay, in which MICs for cefepime $\geq 16 \mu \mathrm{g} / \mathrm{mL}$ and $\geq 2 \mu \mathrm{g} / \mathrm{mL}$, respectively, were considered positive tests for ESBL activity ${ }^{(5)(9)}$.

All the phenotypic tests were performed using fresh cultures. The inoculum was adjusted to the $0.5 \mathrm{McF}$ arland standard, which contains approximately 1 to $2 \times 10^{8}$ colony forming units $(\mathrm{CFU}) / \mathrm{mL}$. This adjustment was necessary because an inoculum-dependent effect is observed in $\beta$-lactamaseproducing samples ${ }^{(10)}$.

A high (56\%) frequency of ESBL gene prevalence was detected from the E. cloacae isolates, and all ESBL-positive samples also tested positive in the CPM2 assay. No correlation was identified between clonal genotypes and the absence or presence of ESBL.

Bell et al. and Park et al. proposed screening methods for ESBL in Enterobacter spp. based on MIC $>0.25 \mu \mathrm{g} / \mathrm{mL}$ and $\mathrm{MIC} \geq 1 \mu \mathrm{g} / \mathrm{mL}$ for cefepime, respectively ${ }^{(11)(12)}$. When applying these criteria to our samples, we did not find similar sensitivity and specificity to these reports. However, when we increased the stringency by raising the cutoff MIC value for cefepime to $\geq 2 \mu \mathrm{g} / \mathrm{mL}$, we obtained $100 \%$ sensitivity and $94 \%$ specificity (Table 1). We therefore conclude that this is the most accurate and easily applicable method for ESBL detection activity in Enterobacter spp.

CPM16 revealed greater (100\%) specificity with reduced $(80 \%)$ sensitivity (Table 1), but use of this method is limited in clinical routines, where the MIC is determined using automated methods. With these methods, $16 \mu \mathrm{g} / \mathrm{mL}$ of cefepime is a concentration not routinely included in dilution panels, whereas $2 \mu \mathrm{g} / \mathrm{mL}$ of cefepime is frequently found.

Jeong et al. achieved $98.4 \%$ sensitivity for ESBL detection in chromosomal AmpC producers using the CABA method ${ }^{(7)}$. This is in contrast with the $62 \%$ sensitivity and $91 \%$ specificity we attained in this study (Table 1).

Although Tzelepi et al. ${ }^{(8)}$ demonstrated that the DDAc method using cefepime worked well in species that possessed a chromosomal ampC gene $^{(8)}$, in this study we achieved only $49 \%$ sensitivity with this method (Table 1). This may have been due to differences in interpretation of the results, which can be subjective. In our case, it was difficult to determine whether the $\beta$-lactam inhibitory zones were qualitatively altered.
TABLE 1 - Sensitivity and specificity of the four different phenotypic methods used for ESBL detection in Enterobacter cloacae isolates.

\begin{tabular}{lcccc}
\hline & \multicolumn{4}{c}{ Methods } \\
\cline { 2 - 5 } & CABA & DDAc & CPM16 & CPM2 \\
\hline Sensitivity (\%) & 64.0 & 50.0 & 80.0 & 100.0 \\
Specificity (\%) & 91.0 & 91.0 & 100.0 & 94.0 \\
\hline
\end{tabular}

ESBL: Extended-spectrum $\beta$ lactamase; CABA: ceftazidime and cefotaxime disks with and without clavulanic acid + boronic acid; DDAc: disk approximation using cefepime and amoxicillin-clavulanic acid; CPM16 and CPM2: MICs for cefepime $\geq 16 \mu \mathrm{g} / \mathrm{mL}$ and $\geq 2 \mu \mathrm{g} / \mathrm{mL}$, respectively. MICs: minimum inhibitory concentrations.

Interpretation of results from this assay likely requires expertise of the analyst. Furthermore, the ideal distance between disks depends on the sensitivity profile of the bacteria, as has been previously reported ${ }^{(13)}$. A modification to this test was recently suggested; adding phenylboronic acid to the cefepime disk would improve detection of ESBL activity in AmpC producing samples ${ }^{(14)}$.

To consider the accuracy of the methods, the CABA and DDAc methods detected three ESBL-like samples that were negative by PCR (Figure 1), while the CPM2 method detected two. The discrepancy may be due to the primer sequences, which were designed to recognize the most common ESBL families (TEM, CTX-M, and SHV). However, there are other, less prevalent families that were not assessed in this study ${ }^{(13)}$. Therefore it is possible that these were not false-positive results from the phenotypic assays, but false-negative results from the PCR-based assay.

In the present study, nine (20\%) samples showed sensitivity to cefepime (MIC between $2 \mu \mathrm{g} / \mathrm{mL}$ and $8 \mu \mathrm{g} / \mathrm{mL}$ ) and were positive to ESBL with both CPM2 and the genotypic method (Figure 1). These cases demonstrate the necessity for a routine clinical use of a rapid and simple method to detect ESBL activity in Enterobacter spp. isolates since cefepime may be the drug of choice for treatment of Enterobacter spp. infections ${ }^{(15)}$.

However further clinical studies are needed to evaluate patients treated with cefepime to assess the efficacy of this cephalosporin drug in the treatment of ESBL-producing Enterobacter spp. infections. In addition, the rapid detection of ESBL isolates would enable infection control practitioners to implement precautions, avoiding further spread of this pathogen in the hospital setting.

In conclusion, we demonstrate here that for those laboratories that use dilution methods to determine antimicrobial sensitivity, adopting the CPM2 method would be very useful for the phenotypic detection of ESBL in samples of Enterobacter spp., while for laboratories that use only the disk-diffusion method, the CABA method is highly effective. Our findings also suggest the need for greater surveillance of ESBL in Enterobacter spp. infections for both improved treatment options and to reduce the risk of wider outbreaks.

\section{ACKNOWLEDGMENTS}

We thank Janet W. Reid for revising the english text. 


\section{CONFLICT OF INTEREST}

The authors declare that there is no conflict of interest.

\section{FINANCIAL SUPPORT}

This work was supported by the Coordenação de Aperfeiçoamento do Pessoal de Ensino Superior (CAPES), Brazil.

\section{REFERENCES}

1. Crowley B, Ratcliffe G. Extended-spectrum $\beta$-lactamases in Enterobacter cloacae: underestimated but clinically significant! J Antimicrob Chemother 2003; 51:1316-1317.

2. Bonnet R. Growing Group of Extended-Spectrum $\beta$-Lactamases: the CTX-M Enzymes. Antimicrob Agents Chemother 2004; 48:1-14.

3. Menegucci TC, Barros JPRP, Costa BB, Tsuneto PY, Aoki EE, Zarpellon MN, et al. Perfil de sensibilidade e prevalência de bacilos Gram-negativos em um hospital ensino. XIII Congresso Brasileiro de Controle de Infecção e Epidemiologia Hospitalar. Anais J Infect Control 2012; 1:199.

4. Silbert S, Pfaller MA, Hollis RJ, Barth AL, Sader HS. Evaluation of three molecular typing techniques for nonfermentative Gramnegative bacilli. Infect Control Hosp Epidemiol 2004; 25:847-851.

5. Clinical and Laboratory Standards Institute (CLSI). Performance Standards for Antimicrobial Susceptibility Testing. Twentieth Informational Supplement. Approved Standart M100-S23. Wayne, PA: CLSI; 2013.

6. Monstein HJ, Ostholm-Balkhed A, Nilsson MV, Nilsson M, Dornbusch K, Nilsson LE. Multiplex PCR amplification assay for the detection of blaSHV, blaTEM and blaCTX-M genes in Enterobacteriaceae. Apmis 2007; 115:1400-1408.
7. Jeong SH, Song W, Park MJ, Kim JS, Kim HS, Bae IK, et al. Boronic acid disk tests for identification of extended-spectrum $\beta$-lactamase production in clinical isolates of Enterobacteriaceae producing chromosomal AmpC $\beta$-lactamases. Int J Antimicrob Agents 2008; 31:467-471.

8. Tzelepi E, Giakkoupi P, Sofianou D, Loukova V, Kemeroglou A, Tsakris A. Detection of Extended-Spectrum $\beta$-Lactamases in Clinical Isolates of Enterobacter cloacae and Enterobacter aerogenes. J Clin Microbiol 2000; 38:542-546.

9. European Committee on Antimicrobial Susceptibility Testing (EUCAST). Breakpoint tables for interpretation of MICs and zone diameters. Version 2.0. [Cited 2014 October 2]. EUCAST; 2013. Available at (http://www.eucast.org/clinical_breakpoints).

10. Maglio D, Ong C, Banevicius MA, Geng Q, Nightingale $\mathrm{CH}$, Nicolau DP. Determination of the in vivo pharmacodynamic profile of cefepime against extended-spectrum- $\beta$-lactamaseproducing Escherichia coli at various inocula. Antimicrob Agents Chemother 2004; 48:1941-1947.

11. Bell JM, Turnidge JD, Jones RN, SENTRY Asia-Pacific Participants. Prevalence of Extended-Spectrum $\beta$-LactamaseProducing Enterobacter cloacae in the Asia-Pacific Region: Results from the SENTRY Antimicrobial Surveillance Program, 1998 to 2001. Antimicrob Agents Chemother 2003; 47:3989-3993.

12. Park YJ, Park SY, Oh EJ, Park JJ, Lee KY, Woo GJ, Lee $\mathrm{K}$. Occurrence of extended-spectrum $\beta$-lactamases among chromosomal AmpC-producing Enterobacter cloacae, Citrobacter freundii, and Serratia marcescens in Korea and investigation of screening criteria. Diagn Microbiol Infect Dis 2005; 51:265-269.

13. Oliveira KRP, Barth AL. $\beta$-lactamases na família Enterobacteriaceae: métodos de deteç̧ão e prevalência. Rio Grande do Sul: UFRGS; 2008. p. 89.

14. Willems E, Verhaegen J, Magerman K, Nys S, Cartuyvels R. Towards a phenotypic screening strategy for emerging $\beta$-lactamases in Gram-negative bacilli. Int J Antimicrob Agents 2013; 41:99-109.

15. Endimiani A, Perez F, Bonomo RA. Cefepime: a reappraisal in an era of increasing antimicrobial resistance. Expert Rev Anti Infect Ther 2008; 6:805-824. 